\title{
Denumerants of 3-numerical semigroups
}

\author{
Aguiló-Gost F. ${ }^{1 \star}$, García-Sánchez P. ${ }^{2 \star \star}$, and Llena D. ${ }^{3 \star \star \star}$ \\ 1 Departament MA-IV. Universitat Politècnica de Catalunya. matfag@ma4.upc.edu \\ 2 Departamento de Álgebra. Universidad de Granada. pedro@ugr.es \\ 3 Departamento de Matemáticas. Universidad de Almería. dllena@ual.es
}

\begin{abstract}
Denumerants of numerical semigroups are known to be difficult to obtain, even with small embedding dimension of the semigroups. In this work we give some results on denumerants of 3 -semigroups $S=\langle a, b, c\rangle$. The time efficiency of the resulting algorithms range from $O(1)$ to $O(c)$. Closed expressions are obtained under certain conditions.
\end{abstract}

Key words: Denumerant, L-shapes, numerical semigroup, factorization.

\section{Introduction}

Let $\mathbb{N}_{0}$ be the set of non negative integers. Given a set $A=\left\{a_{1}, \ldots, a_{n}\right\} \subset \mathbb{N}_{0}$, $\operatorname{gcd}\left(a_{1}, \ldots, a_{n}\right)=1$, the $n$-numerical semigroup $S=S(A)$ generated by $A$ is defined by $\left\langle a_{1}, \ldots, a_{m}\right\rangle=\left\{x_{1} a_{1}+\cdots+x_{n} a_{n}: x_{1}, \ldots, x_{n} \in \mathbb{N}_{0}\right\}$. If $A$ is a minimal set of generators then $S$ has embedding dimension e $(S)=n$. An element $m \in S$ has a factorization $\left(t_{1}, \ldots, t_{n}\right)$ in $S$ if $m=t_{1} a_{1}+\cdots+t_{n} a_{n}$. The set of factorizations of $m$ in $S$ is denoted by $\mathcal{F}(m, S)=\left\{\left(x_{1}, \ldots, x_{n}\right) \in\right.$ $\left.\mathbb{N}_{0}^{n}: x_{1} a_{1}+\cdots+x_{n} a_{n}=m\right\}$. The denumerant of $m$ in $S$ is the cardinality $\mathrm{d}(m, S)=|\mathcal{F}(m, S)|$.

In this work we give some results on denumerants of generic elements of embedding dimension three numerical semigroups $S=\langle a, b, c\rangle$. Algorithms of time-cost ranging from $O(1)$ to $O(c)$ (in the worst case) are also derived. We use minimum distance diagrams related to $S$ as a main tool. In particular, we use the main results given in [1].

\footnotetext{
* Work supported by the CICYT and the 'European Regional Development Fund' under project MTM2011-28800-C02-01 and Agència de Gestió d'Ajuts Universitaris i de Recerca under project 2009SGR1387.

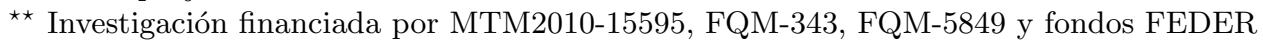

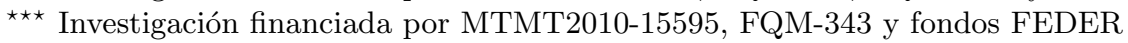




\section{Tools}

From now on we denote the equivalence class of $n$ modulo $N$ as $[n]_{N}$. Given $m \in S \backslash\{0\}$, the Apéry set of $m$ in $S$ is $\operatorname{Ap}(m, S)=\{s \in S: s-m \notin S\}$. This set encodes many properties of semigroup. It can be shown that $|\operatorname{Ap}(m, S)|=$ $m$ and $\operatorname{Ap}(m, S)=\left\{l_{0}, \ldots, l_{m-1}\right\}$ with $l_{k} \in[k]_{m}$ for $0 \leq k<m$.

Minimum Distance Diagrams, $\mathcal{H}$, of numerical semigroups are used to study many distance-related and distribution of the semigroup elements. The minimum distance diagrams related to $S=\left\langle a_{1}, \ldots, a_{n}\right\rangle$ are sets of cardinality $|\mathcal{H}|=a_{n}$ of unitary cubes $\llbracket i_{1}, \ldots, i_{n-1} \rrbracket=\left[i_{1}, i_{1}+1\right] \times \cdots \times\left[i_{n-1}, i_{n-1}+1\right]$ in the first orthant of $\mathbb{R}^{n-1}$ with coordinates $\left(i_{1}, \ldots, i_{n-1}\right) \in \mathbb{N}_{0}^{n-1}$ representing factorizations of the elements of $\operatorname{Ap}\left(a_{n}, S\right)$, where $[s, t]=\{r \in \mathbb{R}: s \leq r \leq t\}$. Each element $l_{k} \in \operatorname{Ap}\left(a_{n}, S\right)$ is represented by exactly one cube $\llbracket i_{1}, \ldots, i_{n-1} \rrbracket$ in $\mathcal{H}$ with $i_{1} a_{1}+\cdots+i_{n-1} a_{n-1}=l_{k}$. Given any $m \in S$, with $m \in\left[l_{k}\right]_{a_{n}}$, we call the basic coordinates of $m$ with respect to $\mathcal{H}$ to $\left(x_{1}, \ldots, x_{n-1}\right) \in \mathbb{N}_{0}^{n-1}$ if $\llbracket x_{1}, \ldots, x_{n-1} \rrbracket \in \mathcal{H}$ and $x_{1} a_{1}+\cdots+x_{n-1} a_{n-1}=l_{k}$. We also call the basic factorization of $m$ in $S$ with respecto to $\mathcal{H}$ to $\left(x_{1}, \ldots, x_{n-1}, \frac{m-l_{k}}{a_{n}}\right) \in \mathcal{F}(m, S)$.

These diagrams were used in [1] to study some aspects on factorization and catenary degree of 3-numerical semigroups $S=\langle a, b, c\rangle$. In particular, we recall here some nomenclature and results. Diagrams related to $S$ are Lshaped or rectangles (considered as degenerated L-shapes with $w y=0$ ) and are denoted by $\mathcal{H}=\mathrm{L}(l, h, w, y)$, where these entries are the lengths of the sides $0 \leq w<l, 0 \leq y<h, \operatorname{gcd}(l, h, w, y)=1$ and $l h-w y=c$. The diagram $\mathcal{H}$ tessellates $\mathbb{R}^{2}$ by translation through the vectors $\boldsymbol{u}=(l,-y)$ and $\boldsymbol{v}=(-w, h)$. See $[2,3]$ for more details.

For the parameters $\delta=(l a-y b) / c$ and $\theta=(h b-w a) / c$, we have $\delta \geq 0$, $\theta \geq 0, \delta+\theta>0, \delta+y>0$ and $\theta+w>0$ whenever $\mathcal{H}=\mathrm{L}(l, h, w, y)$ is a minimum distance diagram of $S=\langle a, b, c\rangle$.

Theorem 1 ([1, Th. 2]). Given $\mathcal{H}=\mathrm{L}(l, h, w, y)$ a minimum distance diagram of $S=\langle a, b, c\rangle$ and $m \in S$, let $\left(x_{0}, y_{0}, z_{0}\right)$ be the basic factorization of $m$ in $S$ respect to $\mathcal{H}$, then

$$
\mathrm{d}(m, S)=1+\left\lfloor\frac{z_{0}}{\delta+\theta}\right\rfloor+\sum_{k=0}^{\left\lfloor\frac{z_{0}}{\delta+\theta}\right\rfloor}\left(S_{k}+T_{k}\right),
$$

with

$$
S_{k}= \begin{cases}\left\lfloor\frac{y_{0}+k(h-y)}{y}\right\rfloor & \text { if } \delta=0, \\ \left\lfloor\frac{z_{0}-k(\delta+\theta)}{\delta}\right\rfloor & \text { if } y=0, \\ \min \left\{\left\lfloor\frac{y_{0}+k(h-y)}{y}\right\rfloor,\left\lfloor\frac{z_{0}-k(\delta+\theta)}{\delta}\right\rfloor\right\} & \text { if } \delta y \neq 0,\end{cases}
$$

and 


$$
T_{k}= \begin{cases}\left\lfloor\frac{x_{0}+k(l-w)}{w}\right\rfloor & \text { if } \theta=0, \\ \left\lfloor\frac{z_{0}-k(\delta+\theta)}{\theta}\right\rfloor & \text { if } w=0, \\ \min \left\{\left\lfloor\frac{x_{0}+k(l-w)}{w}\right\rfloor,\left\lfloor\frac{z_{0}-k(\delta+\theta)}{\theta}\right\rfloor\right\} & \text { if } \theta w \neq 0 .\end{cases}
$$

This theorem does not give an efficient algorithm for computing denumerants. The reason is not the expression of minima appearing in $S_{k}$ when $\delta y \neq 0$ or in $T_{k}$ when $\theta w \neq 0$, these minima can be left out for $k \geq\left[\frac{y z_{0}-\delta y_{0}}{\delta(h-y)+y(\delta+\theta)}\right]$ in $S_{k}$ when $\delta y \neq 0$ and for $k \geq\left\lceil\frac{w z_{0}-\theta x_{0}}{\theta(l-w)+w(\delta+\theta)}\right\rceil$ in $T_{k}$ when $\theta w \neq 0$. The reason is the expression of the sum. Essentially we have to compute sums like

$$
\sum_{k=0}^{N}\left\lfloor\frac{\alpha \pm k \beta}{q}\right\rfloor=\sum_{k=0}^{N}(\bar{\alpha} \pm k \bar{\beta})+\sum_{k=0}^{N}\left\lfloor\frac{s \pm k t}{q}\right\rfloor,
$$

for $\alpha, \beta, \bar{\alpha}, \bar{\beta}, q \in \mathbb{N}$, with $q \neq 0, \alpha=\bar{\alpha} q+s, 0 \leq s<q$, and $\beta=\bar{\beta} q+t$, $0 \leq t<q$.

\section{Trying $\sum_{k=0}^{N}\left\lfloor\frac{s \pm k t}{q}\right\rfloor$}

The efficiency of computing denumerants using these sums is compromised because of the number of terms to be added $N, O(N)=O\left(z_{0}\right)$. The coordinate $z_{0}$ of a basic factorization can become very large. Thus, we need to save add up all those terms.

In this section we try the $\operatorname{sum} \sum_{k=0}^{N}\left\lfloor\frac{s+k t}{q}\right\rfloor$ with $0 \leq s, t<q$. The sum with the minus sign can be solved using an analogous method by symmetry. The idea of the method we propose here is making this addition as a Lebesguelike discrete integration. Let us consider the graph of the function $f(x)=$ $\left\lfloor\frac{s+t x}{q}\right\rfloor$ and pay attention to the constant parts of the graph. Let us denote the interval $I_{m}=\left[x_{m}, x_{m+1}\right)$ where de function $f$ has constant value $m$, that is $x_{m}=\frac{m q-s}{t}$ (if $t=0$ the entire sum equals zero). Then, some main properties hold:

(1) There are $M+1$ different intervals, with $M=\left\lfloor\frac{s+N t}{q}\right\rfloor$. Each interval $I_{m}$ has length $x_{n+1}-x_{m}=\frac{q}{t}$ except, possibly, $I_{0}$ and/or $I_{M}$.

(2) $\left\lfloor\frac{q}{t}\right\rfloor \leq\left|I_{m} \cap \mathbb{N}\right| \leq\left\lceil\frac{q}{t}\right\rceil$ except, possibly, $I_{0}$ and/or $I_{M}$.

(3) Those intervals $I_{m}$ with $x_{m} \in \mathbb{N}_{0}$ are iS-type intervals. Intervals with $\left|I_{m} \cap \mathbb{N}\right|=\left\lceil\frac{q}{t}\right\rceil$ are $\mathrm{mS}$-type intervals. Intervals iS are also $\mathrm{mS}$ except, possibly, $I_{M}$. There can be $\mathrm{mS}$-intervals that are not iS-intervals.

(4) The behaviour of intervals at the same relative position between two consecutive iS-intervals is the same. There are $t-1$ intervals between two 
consecutive iS-intervals. The behaviour of intervals $I_{m}$ is periodic of period $t$. Depending on the values $s, t$ and $q$, this period is not the minimum one.

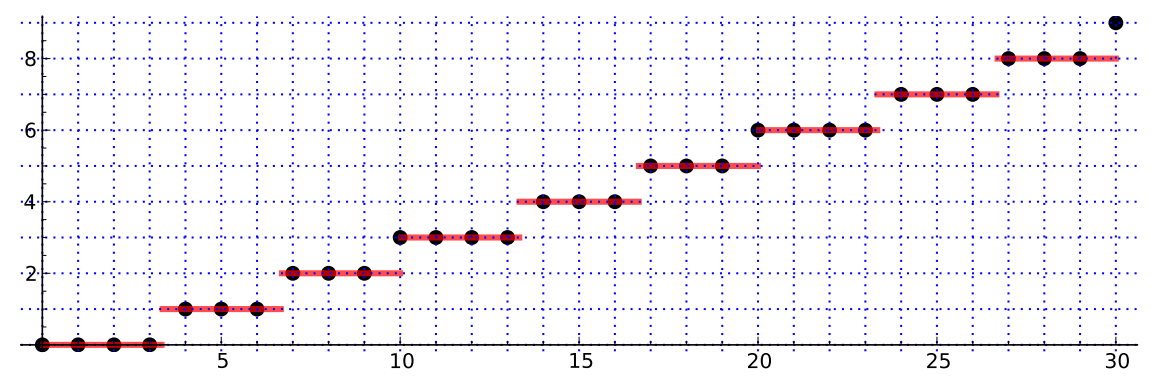

Fig. 1. Graph of $f$ with $s=0, t=3, q=10$

Figure 1 shows the graph of $f$ with parameters $s=0, t=3$ and $q=10$. Note the 3 -periodic behaviour of the intervals $I_{m}, m \in\{0, \ldots, 9\}$. In this case, only the iS-intervals contain $\left\lceil\frac{q}{t}\right\rceil=4$ integral values, except $I_{M}$ (with $M=9$ ) for $N=30$ that is only one integral point $I_{9}=\{9\}$.

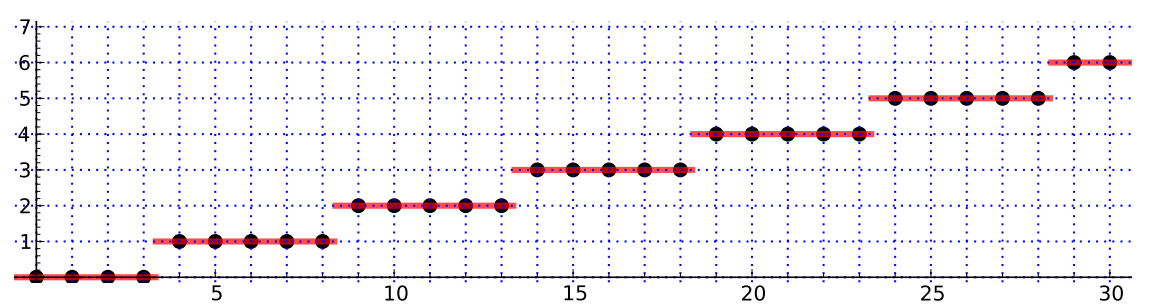

Fig. 2. Graph of $f$ with $s=5, t=3, q=15$

We study two main cases, $\operatorname{gcd}(t, q)>1$ and $\operatorname{gcd}(t, q)=1$. Figure 1 is the simple behaviour of the second case. The first case can be studied for $t \mid q$ and $t \nmid q$. When $t \mid q$, all intervals have the same behavior except, possibly, the first and the last ones; this is the case of Figure 2. Note that all intervals are of type $\mathrm{mS}$ and there is no iS-intervals, essentially the existence of iS-intervals depends on the value $s$. When $t \nmid q$ and $\operatorname{gcd}(t, q)=g>1$, depending on the value of $s$ appear iS-intervals and the period is not $t$ but $t / g$; Figure 3 shows this case.

Depending on the behavior of intervals $I_{m}$ the value of the sum can be arranged in an almost closed expression that sometimes results in a closed expression. The following results take into account all cases (that are more than the examples appearing in the three figures mentioned above).

From now on set $M=\left\lfloor\frac{s+N t}{q}\right\rfloor$ and $x_{M}=\frac{M q-s}{t}$. 


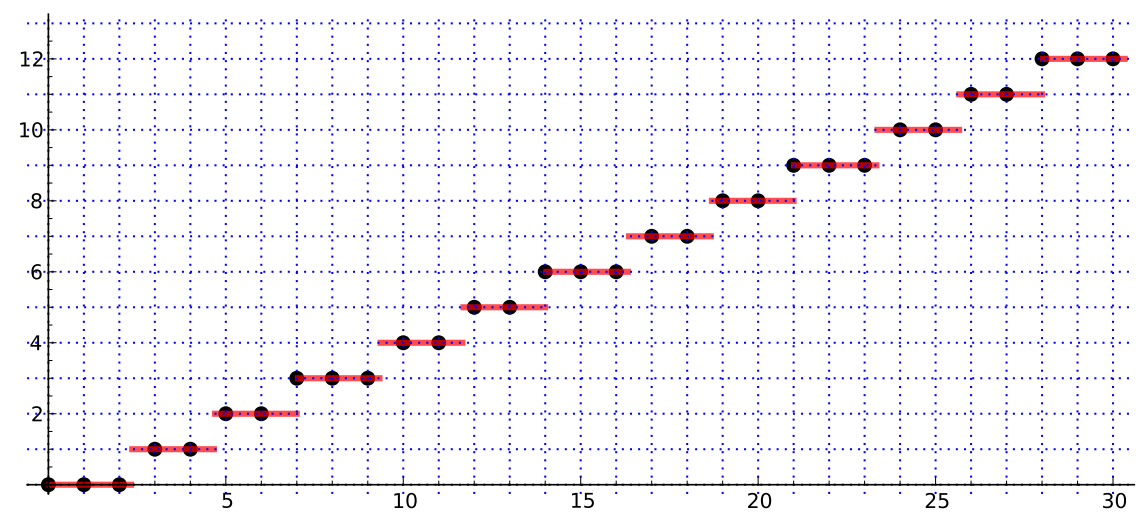

Fig. 3. Graph of $f$ with $s=0, t=6, q=14$

Theorem 2 (Case $t \mid q)$. Assume $q=\bar{q} t$. Then,

$$
\sum_{k=0}^{N}\left\lfloor\frac{s+k t}{q}\right\rfloor=\bar{q} \frac{M(M-1)}{2}+M\left(N-\left\lceil x_{M}\right\rceil+1\right) .
$$

Theorem 2 is a simple case for applying the Lebesgue-like discrete summation. Thi theorem give closed expressions for denumerants.

Now we give the cases $t \nmid q$ with $\operatorname{gcd}(t, q)=1 \operatorname{or} \operatorname{gcd}(t, q)=g>1$. To describe it we must define another type of intervals. Define $\hat{s}$ and $\hat{q}$ by

$$
q=\bar{q} t+\hat{q}, s=\bar{s} t+\hat{s}, \quad 0 \leq \hat{s}, \hat{q}<t
$$

We say that $I_{m}$ is an hS-interval if $(\hat{s}-m \hat{q})(\bmod t)<\hat{q}$. We denote the sets $I=$ $\left\{i_{1}, \ldots, i_{\alpha}\right\}, J=\left\{j_{1}, \ldots, j_{n}\right\}, K=\left\{k_{1}, \ldots, k_{v}\right\}$ of ordered indices of all hStype intervals taken from some region to be defined. We also denote the sums $S_{I}=\sum_{l=1}^{\alpha} i_{l}, S_{J}=\sum_{l=1}^{n} j_{l}, S_{K}=\sum_{l=1}^{v} k_{l}, S=\bar{q} \frac{(M-1) M}{2}+M\left(N-\left\lceil x_{M}\right\rceil+1\right)$ and $\mathbb{S}=\sum_{k=0}^{N}\left\lfloor\frac{s+k t}{q}\right\rfloor$.

Theorem 3 (Case $t \nmid q$ and $\operatorname{gcd}(t, q)=1)$. Set $m_{0} \equiv q^{-1} s(\bmod t)$ with $m_{0} \in\{0, \ldots, t-1\}$ and $u=\left\lfloor\frac{M-m_{0}-1}{t}\right\rfloor$.

(a) If $m_{0}=0$ and $u \leq 0$, or $m_{0}=M$, take $K \subset[1, M-1]$. Then $\mathbb{S}=S+S_{K}$.

(b) If $m_{0}=0$ and $u>0$, take $J \subset[0, t-1]$ and $K \subset[u t, M-1]$. Then

$\mathbb{S}=S+u S_{J}+n t \frac{(u-1) u}{2}+S_{K}$.

(c) If $0<m_{0}<M$ and $m_{0}+t \geq M$, take $I \subset\left[1, m_{0}-1\right]$ and $K \subset\left[m_{0}, M-1\right]$.

Then $\mathbb{S}=S+S_{I}+S_{K}$.

(d) If $0<m_{0}<M$ and $m_{0}+t<M$, take the sets $I \subset\left[1, m_{0}-1\right]$ and

$J \subset\left[m_{0}, m_{0}+t-1\right]$. Take also $K \subset\left[m_{0}+u t, M-1\right]$ whenever $u>0$ and

$K=\emptyset$ otherwise (thus $S_{K}=0$ ). Then $\mathbb{S}=S+S_{I}+u S_{J}+n t \frac{(u-1) u}{2}+S_{K}$. 
Theorem 4 (Case $t \nmid q$ and $\operatorname{gcd}(t, q)=g>1)$. Set $\tilde{t}=t / g$. Take $J \subset$ $[0, \tilde{t}-1]$. Set $m_{0}=j_{1}$ and $u=\left\lfloor\frac{M-m_{0}-1}{\tilde{t}}\right\rfloor$.

(a)If $m_{0} \geq M$, then $\mathbb{S}=S$.

(b) If $0 \leq m_{0}<M$, take $K \subset\left[m_{0}+u \tilde{t}, M-1\right]$. Then $\mathbb{S}=S+u S_{J}+n \tilde{t} \frac{(u-1) u}{2}+$ $S_{K}$.

Theorems 3 and 4 do not give closed expressions for denumerants because of the computation of the sets of indices. This task has order $O(t)$ in the worst case. However, the resulting algorithm is efficient. Similar results can be obtained to compute the sum $\sum_{k=0}^{N}\left\lfloor\frac{s-k t}{q}\right\rfloor$.

\section{Application example}

Each 3-semigroup has its own denumerant's information encoded in the related L-shapes. As an example, let us consider for instance $S=\langle 121,1111,2323\rangle$ with related L-shape $\mathrm{L}(101,23,0,11)$. Thus, $\delta=0$ and $\theta=25553$.

This semigroup belongs to the general case $\delta=w=0$, according with Theorem 1 (there are exactly five generic cases $\{\delta=0, w=0\},\{\delta=0, w \neq 0\}$, $\{\theta=0, y=0\},\{\theta=0, y \neq 0\}$ and $\{\delta \neq 0, \theta \neq 0\})$. Let us consider the case $\{\delta=0, w=0\}$ (then $y \theta \neq 0$ ). Assume $S=\langle a, b, c\rangle$ and $\mathcal{H}=\mathrm{L}(l, h, 0, y)$ belongs to this case. Then, the denumerant is given by

$$
\mathrm{d}(m, S)=1+\left\lfloor\frac{z_{0}}{\theta}\right\rfloor+\sum_{k=0}^{\left\lfloor\frac{z_{0}}{\theta}\right\rfloor}\left(\left\lfloor\frac{y_{0}+k(h-y)}{y}\right\rfloor+\left\lfloor\frac{z_{0}-k \theta}{\theta}\right\rfloor\right),
$$

where $\left(x_{0}, y_{0}, z_{0}\right)$ is the basic factorization of $m \in S$ with respect to $\mathcal{H}$. Set $y_{0}=\overline{y_{0}} y+\hat{y_{0}}$ and $h-y=\bar{n} y+\hat{n}$ with $0 \leq \hat{y}_{0}, \hat{n}<y$. Then,

$$
\sum_{k=0}^{\lambda_{0}}\left\lfloor\frac{y_{0}+k(h-y)}{y}\right\rfloor=\left(1+\lambda_{0}\right)\left[\overline{y_{0}}+\frac{1}{2} \lambda_{0} \hat{n}\right]+\sum_{k=0}^{\lambda_{0}}\left\lfloor\frac{\hat{y}_{0}+k \hat{n}}{y}\right\rfloor,
$$

with $\lambda_{0}=\left\lfloor\frac{z_{0}}{\theta}\right\rfloor$. Similarly,

$$
\sum_{k=0}^{\lambda_{0}}\left\lfloor\frac{z_{0}-k \theta}{\theta}\right\rfloor=\sum_{k=0}^{\lambda_{0}}\left(\lambda_{0}-k\right)=\frac{1}{2} \lambda_{0}\left(1+\lambda_{0}\right) .
$$

Therefore, the denumerant is given by

$$
\mathrm{d}(m, S)=\left(1+\lambda_{0}\right)\left[1+\overline{y_{0}}+\frac{1}{2} \lambda_{0}(1+\hat{n})\right]+\sum_{k=0}^{\lambda_{0}}\left\lfloor\frac{\hat{y}_{0}+k \hat{n}}{y}\right\rfloor .
$$

For $S=\langle 121,1111,2323\rangle$, let us take the family of elements of $m_{t} \in S$ given by the basic factorization $\left(x_{0}, y_{0}, z_{0}\right)=(1,1, t)$, i.e. $m_{t}=1232+2323 t$. Then, 
in this case, the parameters are $\overline{y_{0}}=0, \hat{y_{0}}=1, \bar{n}=1, \hat{n}=1, y=11$ and $\lambda_{0}=\left\lfloor\frac{t}{11}\right\rfloor$. Thus, the denumerant is

$$
\mathrm{d}\left(m_{t}, S\right)=\left(1+\left\lfloor\frac{t}{11}\right\rfloor\right)^{2}+\sum_{k=0}^{\left\lfloor\frac{t}{11}\right\rfloor}\left\lfloor\frac{1+k}{11}\right\rfloor .
$$

Applying the results given in Section 2, we can take large values for $t$. For instance, when $t=10^{10^{3}}$, the denumerant $\mathrm{d}\left(m_{t},\langle 121,1111,2323\rangle\right)$ is

86401202103681442373725632370418508815236113947008384865900 18355482581937070575687706067297170372656872124127106355013 83705399205261030784757611250382814450458614863741996576336 04381350933152481314521762235166717566155055157623293969446 08615786367662651758827936885013572929910812562936838277177 28615652451360231647622463525737529205087066732129366369694 10298518906363268082467489122400475776673691012914136765846 09475113507696144835778060008209708220861265294901965031701 37343295778759267316777895472559376756325707720000071855742 17322046447831982160259072389696123371066939277711855383683 57767721175049376552251604388714812532134415445726788119363 68575850635618156081169145034427047492856768128250632899016 17212129580044803105058702050223244750468912218659498851826 79421268043289617415276449211375778795179442471940300299127 63714373681696491261302114173003214221470476571235948015220 40805924474753184661427823025445679496557123444293037103918 92419497358929307066648456735251843730273406430491864133064 41135539234447721104466978180270005635128920808812849905257 02863506089428812738102330203742063649097015260203032359621 37368659029328436873147224837998307789261605573480576796432 62618991722039200052628030197744818517237285777788689442446 19935553020251798305234674292358662883696542490554205560718 08819266153700438284637594415248278846875002464266544621032 88133854180289115673176777959791111114837655293948229185572 44097229517541555204729647852029427426683172559095558399454 98778302979322010609690412121408608231615838397400125843956 68593577168522246218425317381671565212196629533706324699481 36819653825790303884550101940617170942701351142361553525485 92011247720180542800904885687437465972185292562403508661788 89888201723589125565572218910631240914633142217355801591441 56915987081038318091955413538125941123771789975049999096223 62687036855313366690934052679792085475244714662187442952179 12806317148265826264562297662155082803832709199101981830544 850830347007054848234787152293363588331442400219780 
8 Aguiló-Gost F., García-Sánchez P., and Llena D.

\section{References}

[1] F. Aguiló-Gost and P.A. García-Sánchez. Factoring in embedding dimension three numerical semigroups, The Electronical Journal of Combinatorics, 17, R138: 21 pp. 2010.

[2] M.A. Fiol, J.L.A. Yebra, I. Alegre and M.Valero. A discrete optimization problem in local networks and data alignment, IEEE Transaction of Computations, C36:702-713, 1987.

[3] Ø.J. Rødseth, Weighted multi-connected loop networks, Discrete Mathematics 148:161-173, 1996.

[4] J. C. Rosales and P. A. García-Sánchez. Numerical Semigroups, Developments in Mathematics 20. Springer, New York, 2009. 